\title{
Inhibition of heart transplant injury and graft coronary artery disease after prolonged organ ischemia by selective protein kinase $\mathrm{C}$ regulators
}

\author{
Masashi Tanaka, MD, ${ }^{a}$ Feny Gunawan, ${ }^{\text {a }}$ Raya D. Terry, ${ }^{a}$ Koichi Inagaki, MD, PhD, ${ }^{\mathrm{b}}$ Anthony D. Caffarelli, MD, ${ }^{a}$
} Grant Hoyt, ${ }^{a}$ Philip S. Tsao, PhD, ${ }^{c}$ Daria Mochly-Rosen, PhD, ${ }^{b}$ and Robert C. Robbins, MD ${ }^{\mathrm{a}}$

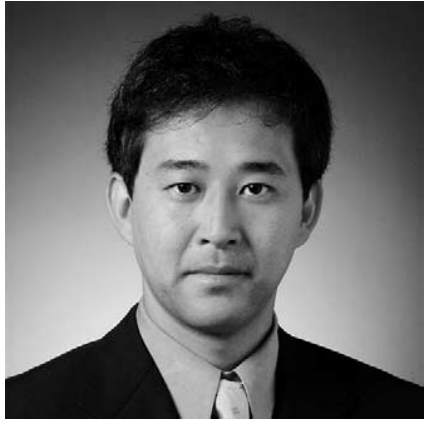

Dr Tanaka

From the Departments of Cardiothoracic Surgery, ${ }^{\mathrm{a}}$ Molecular Pharmacology, ${ }^{\mathrm{b}}$ and Cardiovascular Medicine, ${ }^{\mathrm{c}}$ Stanford University Medical Center, Stanford, Calif.

Supported by National Institutes of Health grants HL65669 (R.C.R.) and HL52141 (D.M.-R.).

D.M.-R. is a founder of KAI Pharmaceuticals, the goal of which is to bring peptide regulators of $\mathrm{PKC}$ to the clinic. The research described in this study, however, was performed in collaboration with her laboratory at the university with sole support from the National Institutes of Health to her university activities.

Received for publication June 17, 2004; revisions received Sept 8, 2004; accepted for publication Sept 20, 2004

Address for reprints: Masashi Tanaka, MD, Department of Cardiothoracic Surgery, Stanford University School of Medicine, Falk Cardiovascular Research Center, Stanford, CA 94305 (E-mail: masahi@ omiya.jichi.ac.jp).

J Thorac Cardiovasc Surg 2005;129:1160-7

$0022-5223 / \$ 30.00$

Copyright (C) 2005 by The American Association for Thoracic Surgery

doi:10.1016/j.jtcvs.2004.09.015
Objective: Transplanted hearts subjected to prolonged ischemia develop ischemiareperfusion injury and graft coronary artery disease. To determine the effect of $\delta$-protein kinase $\mathrm{C}$ and $\epsilon$-protein kinase $\mathrm{C}$ on ischemia-reperfusion injury and the resulting graft coronary artery disease induced by prolonged ischemia, we used a $\delta$-protein kinase $\mathrm{C}$-selective inhibitor peptide and an $\epsilon$-protein kinase $\mathrm{C}$-selective activator peptide after 30 or 120 minutes of ischemia.

Methods: Hearts of piebald viral glaxo (PVG) rats were heterotopically transplanted into allogeneic August Copenhagen Irish (ACI) rats. After cardioplegic arrest of the donor heart, $\epsilon$-protein kinase $\mathrm{C}$ activator was injected antegrade into the coronary arteries. Hearts were procured and bathed in $\epsilon$-protein kinase $\mathrm{C}$ activator, and before reperfusion, $\delta$-protein kinase $\mathrm{C}$ inhibitor was injected into the recipient inferior vena cava. Controls were treated with saline. To analyze ischemia-reperfusion injury, grafts were procured at 4 hours after transplantation and analyzed for superoxide generation; myeloperoxidase activity; tumor necrosis factor $\alpha$, interleukin $1 \beta$, and monocyte/macrophage chemoattractant protein 1 production; and cardiomyocyte apoptosis by terminal deoxynucleotidyl transferase-mediated dUTP nick end labeling and caspase 2, 3, 8, and 9 activity. To analyze graft coronary artery disease, another set of animals underwent equal ischemic times and treatment strategies and then after 90 days were analyzed for graft coronary artery disease indexes.

Results: All measures of ischemia-reperfusion injury and graft coronary artery disease after 120 minutes of ischemia in the saline-treated group were significantly increased relative to those observed after 30 minutes of ischemia. It is important to note that all ischemia-reperfusion injury parameters and graft coronary artery disease indexes decreased significantly in the protein kinase $\mathrm{C}$ regulator-treated group in comparison to saline-treated controls; additionally, these values were equivalent to those in saline-treated controls with 30 minutes of ischemia.

Conclusions: Combined treatment with $\epsilon$-protein kinase $\mathrm{C}$ activator and $\delta$-protein kinase $\mathrm{C}$ inhibitor reduces ischemia-reperfusion injury and decreases the resulting graft coronary artery disease induced by prolonged ischemia.

$\mathrm{D}$ espite recent advances in immunosuppressive therapy and in the treatment and diagnosis of posttransplantation infection-induced complications and acute rejection, graft coronary artery disease (GCAD) remains the leading cause of death in patients who survive more than 1 year after cardiac transplantation. ${ }^{1}$ The development of GCAD in heart transplant patients is currently inevitable, and, unlike with typical coronary artery disease, there is no effective treatment other than retransplantation. The pathophysiological processes that contribute to the initiation and propagation of GCAD, although not completely understood, are multifactorial and likely involve both alloantigen-independent and alloantigendependent mechanisms. ${ }^{2}$

Ischemia-reperfusion injury was the strongest alloantigen-independent factor for the subsequent development of GCAD in a clinical case-control study. ${ }^{3}$ Wang and colleagues ${ }^{4}$ have shown that ischemia-reperfusion injury alone can promote alloantigenindependent formation of GCAD. Ischemia-reperfusion injury generates an inflamma- 
tory environment, which includes the production of the injurious chemokines and cytokines-such as tumor necrosis factor (TNF)- $\alpha$, interleukin (IL)-1 $\beta$, and monocyte/macrophage chemoattractant protein (MCP)-1-and which leads to graft failure. ${ }^{5}$ In addition, numerous studies have shown that cardiomyocyte apoptosis is an early event in cardiac ischemiareperfusion injury, ${ }^{6}$ which led to our hypothesis that early prevention of cardiomyocyte apoptosis should reduce ischemia-reperfusion injury in the transplanted heart and the subsequent development of GCAD.

The protein kinase $\mathrm{C}$ (PKC) family of highly homologous enzymes is involved in a variety of cellular functions. ${ }^{7} \mathrm{We}$ have shown that treatment with an $\epsilon \mathrm{PKC}$-selective activator peptide, $\Psi \in \mathrm{RACK}$ (receptor for activated $\mathrm{C}$ kinase), or a $\delta \mathrm{PKC}$-selective inhibitor peptide, $\delta \mathrm{V} 1-1$, alone conferred cardioprotection to the ischemic heart. ${ }^{8,9}$ We have also shown that combined treatment with these 2 PKC regulator peptides provides greater cardioprotection against ischemia-reperfusion injury than treatment with the individual peptide treatments alone in isolated perfused rat hearts and transgenic mice. ${ }^{10}$ Moreover, we have recently found that these PKC regulating peptides reduced 50-minute ischemia-induced injury and the subsequent development of GCAD in murine cardiac allografts when measured 30 days after transplantation. ${ }^{11}$ In that study, we delivered the $\epsilon \mathrm{PKC}$ activator to the donor 20 minutes before organ harvest, and the $\delta \mathrm{PKC}$ inhibitor was delivered to the recipient abdominal cavity, as opposed to the graft itself. Neither of these delivery routes was likely applicable to the clinical situation. In addition, the effects of these PKC-regulating peptides on ischemia-reperfusion injury and the GCAD induced by prolonged ischemia have not yet been determined. Our hypothesis is that treatment with PKC regulators in a clinically relevant protocol will reduce both immediate ischemia-reperfusion injury and the resulting development of GCAD. Using the previously described selective PKC isozyme regulator peptides, we tested this hypothesis in a rat cardiac allograft model subjected to up to 120 minutes of ischemia and measured the development of GCAD 90 days after transplantation.

\section{Materials and Methods}

Animals

Adult male inbred piebald viral glaxo $\left(\mathrm{PVG} ; \mathrm{RT}^{\mathrm{c}}\right.$ ) and August Copenhagen Irish (ACI; RT1 ${ }^{\mathrm{a}}$ ) rats weighing 200 to $250 \mathrm{~g}$ were purchased from Harlan Sprague-Dawley (Indianapolis, Ind). The PVG rats were used as allograft donors, and the ACI rats were used as recipients. All rats were kept under standard temperature, humidity, and timed-lighting conditions and were provided rat chow and water ad libitum. Animals were housed and cared for in compliance with the Guide for the Care and Use of Laboratory Animals prepared by the Institute of Laboratory Animal Resources, National Research Council, and published by the National Academy Press (revised 1996).

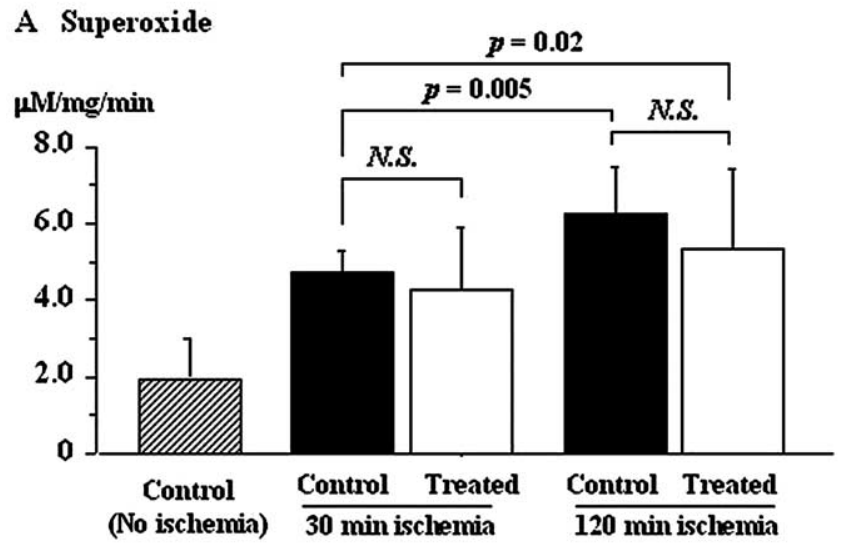

\section{B ISOL TUNEL}

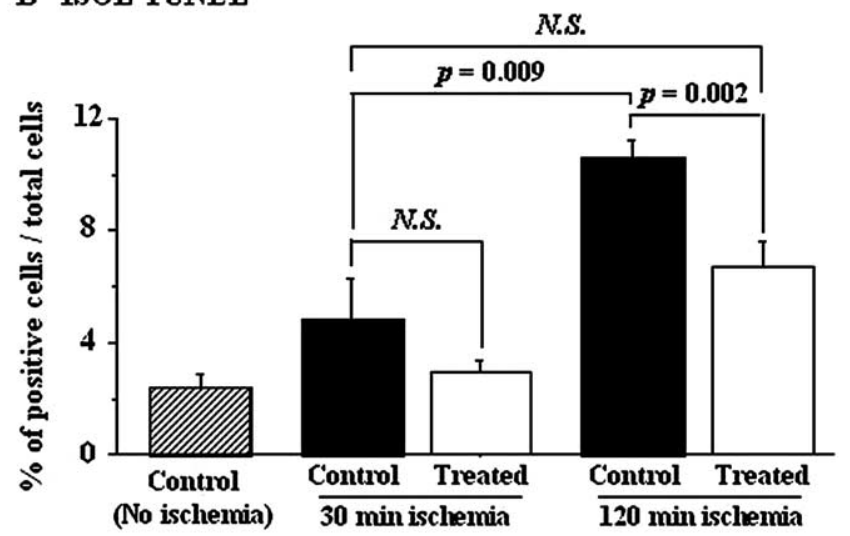

Figure 1. Selective PKC regulators inhibit cardiomyocyte apoptosis, but not superoxide radical production, induced by ischemiareperfusion injury to transplanted hearts subjected to 30 or 120 minutes of ischemia. A, Superoxide production. B, In situ oligo ligation (ISOL) TUNEL-positive cell counts. Closed columns, Control group ( $n=6$ for each ischemic time); open columns, PKC regulator-treated group ( $n=6$ for each ischemic time); N.S., not significant. Values obtained from naive hearts of PVG rats (control; no ischemia) served as a reference $(n=4)$. Mean \pm SE values are shown.

\section{Heterotopic Cardiac Transplantation}

Donor PVG hearts were heterotopically transplanted into the abdomen of ACI recipients as previously described by Ono and Lindsey. ${ }^{12}$ Briefly, the donor heart was induced into cardiac arrest by injection of ice-cold high-potassium cardioplegia solution into the aortic root. The procured hearts were allowed to incubate in a bath of cold saline. After the recipient was anesthetized with $2.5 \%$ inhaled isoflurane (Halocarbon Laboratories, River Edge, NJ) and intraperitoneal sodium pentobarbital $40 \mathrm{mg} / \mathrm{kg}$ (Abbott Laboratories, North Chicago, Ill), the donor ascending aorta and pulmonary artery were anastomosed to the infrarenal abdominal aorta and inferior vena cava, respectively. 


\section{Drug Administration}

In the treated groups, after cardioplegic arrest of the donor heart and ligation of the ascending aorta, $2 \mathrm{~mL}$ of $\epsilon \mathrm{PKC}$ activator ( $\Psi \in \mathrm{RACK} ; 1.5 \mathrm{nmol})$ solution was injected antegrade into the coronary artery system. Hearts were then procured and submerged in $\epsilon \mathrm{PKC}$ activator ( $\Psi \in \mathrm{RACK} ; 0.5 \mu \mathrm{mol} / \mathrm{L}$ ) solution for 10 or 100 minutes at $4^{\circ} \mathrm{C}$. Because standard graft implantation averages approximately 20 minutes, total ischemic times were 30 and 120 minutes. Before reperfusion of the donor heart, $1 \mathrm{~mL}$ of $\delta \mathrm{PKC}$ inhibitor $(\delta \mathrm{V} 1-1 ; 30 \mathrm{nmol})$ solution was injected into the recipient inferior vena cava. Control animals were treated with normal saline.

\section{Experimental Groups}

This was a 2-part study. In the first part, indicators of ischemiareperfusion injury were analyzed after 4 hours of reperfusion $(\mathrm{n}=$ 6 for each ischemic time). In the second part, GCAD was evaluated at 90 days ( $n=8$ for each ischemic time). In the latter group, recipients received cyclosporine A $(7.5 \mathrm{mg} / \mathrm{kg}$ oral gavage) on postoperative days 0 to 9 to inhibit acute rejection.

\section{Superoxide Production}

Superoxide levels were measured in excised tissue by the spin trap method after 4 hours of reperfusion. Superoxide accumulation was measured by using conditioned medium supplemented with the spin trapping agent 4-amino-2,2,6,6,-tetramethylpiperidine-1-oxyl (tempamine, Sigma-Aldrich, St Louis, Mo), as previously described. ${ }^{13}$ Electron paramagnetic resonance (EPR) spectra were recorded at room temperature with a spectrometer (model 8400; Resonance Instruments, Skokie, Ill). The EPR signal intensity was quantified by comparing the double integration of the recorded first-derivative EPR peaks of each sample with that of a standard tempamine spin solution. When tempamine reacts with another radical species, such as superoxide, it loses its EPR signal. Thus, the reduction in peak height is directly proportional to the amount of superoxide produced. All measurements were normalized to the protein concentration of each sample as determined by the bicinchoninic acid method (Pierce Chemical, Rockford, Ill).

\section{In Situ Oligo Ligation Terminal Deoxynucleotidyl Transferase-Mediated dUTP Nick End Labeling Analysis}

Apoptotic cardiomyocyte counts in allograft tissues were determined by in situ staining of DNA strand breaks in serial sections of each specimen with the use of an ApopTag in situ oligo ligation kit with oligo A (Intergen, Purchase, NY), as previously described. ${ }^{14}$ Because conventional terminal deoxynucleotidyl transferase-mediated dUTP nick end labeling (TUNEL) assay can detect nonspecific DNA fragmentation due to necrosis, a more specific in situ ligation assay for identifying apoptotic nuclei was used with hairpin oligonucleotide probes. Cardiomyocyte apoptosis was confirmed by double-staining the sections with $\alpha$-sarcomeric actin (Sigma). The number of TUNEL-positive cardiomyocytes in each cardiac allograft was counted manually by 2 investigators (F.G. and R.D.T.) blinded to the experimental conditions. Cells were counted in 6 animals (4 fields each) at $200 \times$ magnification. The percentage of TUNEL-stained cells was re- corded, ie, the number of labeled nuclei divided by the total number of nuclei.

\section{Enzyme-Linked Immunosorbent Assay, Caspase Activity, and Myeloperoxidase Assays}

Snap-frozen myocardial tissue specimens were homogenized in phosphate-buffered saline and centrifuged at $12,000 \mathrm{~g}$ for 20 minutes at $4^{\circ} \mathrm{C}$. The protein concentration of the supernatant was determined by the bicinchoninic acid method, and aliquots were stored at $-80^{\circ} \mathrm{C}$. Intragraft TNF- $\alpha$, IL- $1 \beta$, and MCP-1/ CCL-2 (BioSource International, Camarillo, Calif) and caspase 2,8 , and 9 activity assay kits were obtained from R\&D Systems (Minneapolis, Minn). The caspase 3 activity assay kit was purchased from BD Biosciences (Palo Alto, Calif). Myeloperoxidase (MPO) activity (as units per milligram of total protein) was assessed in the lysates of reperfused cardiac allografts as previously described. ${ }^{15}$

\section{Morphometric Analysis of GCAD}

At 90 days after transplantation, the cardiac grafts were harvested and embedded in paraffin. Elastica van Gieson staining was performed for morphometric analysis of arterial intimal proliferation, which was performed as described previously. ${ }^{16}$ In brief, the neointima, media, and lumen were measured with SPOT Advanced version 3.4.2 software (Diagnostic Instruments, Inc, Sterling Heights, Mich). The neointima was defined as the area bounded by the internal elastic lamina, the media as the region between the internal and external elastic membranes, and the lumen as the clear region in the vessel. Diseased vessels were identified by greater than $10 \%$ luminal narrowing. Multiple sections from the middle of the heart were used for analysis. Middlesized coronary arteries were analyzed (more than 10 arteries for each graft).

\section{Statistical Analysis}

All results are expressed as mean \pm SE. Data were compared, and between-group differences were analyzed by analysis of variance with a post hoc Bonferroni test. Statistical analyses were performed with StatView 5.0 (SAS Institute, Cary, NC).

\section{Results}

Reduction of Cardiomyocyte Apoptosis With

Prolonged Ischemia to the Levels of Those With Short Periods of Ischemia by Treatment With PKC Regulators During Ischemia-Reperfusion Injury

We first measured superoxide production, because myocardial injury after ischemia-reperfusion is mediated by oxygen-derived free radicals such as superoxide anion. ${ }^{17}$ Four hours after reperfusion, we found a 1.3-fold increase in superoxide production in the transplanted hearts after 120 minutes of ischemia as compared with the hearts subjected to a 30-minute ischemia in both the PKC regulator-treated and the control groups. However, similar production of superoxide occurred in both the treatment and control groups (Figure 1, A). Because ischemia-reperfusion injury causes cardiomyocyte apoptosis in the cardiac allografts, ${ }^{6}$ 
A

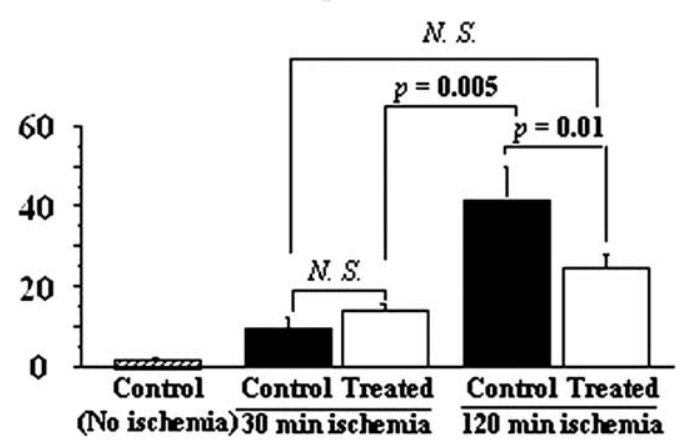

$\mathrm{C}$

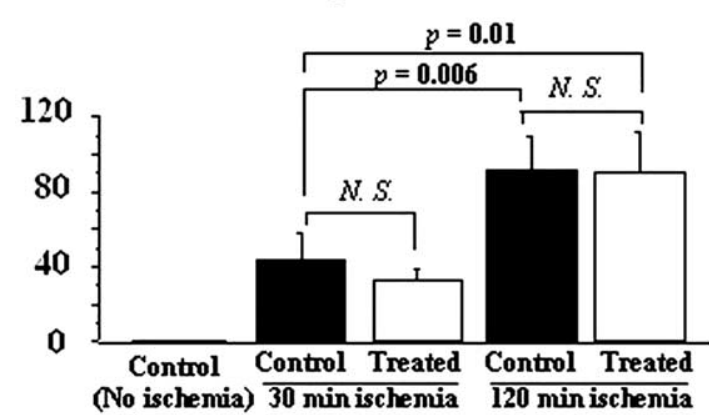

B Caspase-3

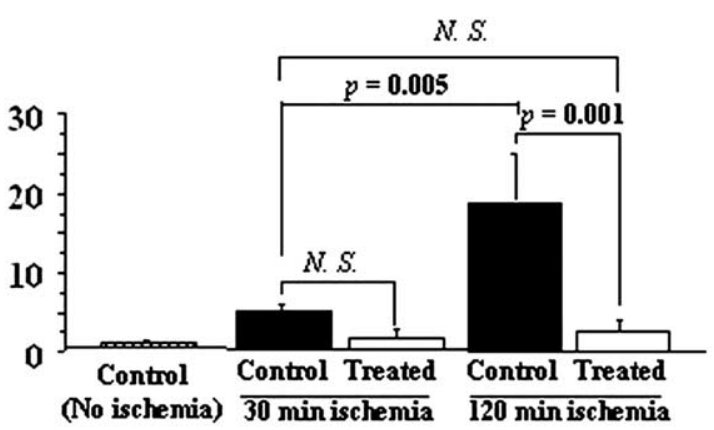

D Caspase-9

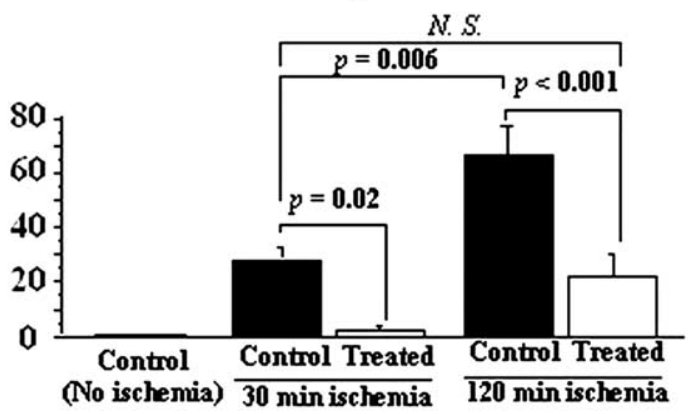

Figure 2. Selective PKC regulators reduce caspase 2, 3, and 9 activity, but not caspase 8 activity, induced by ischemia-reperfusion injury to transplanted hearts subjected to 30 or 120 minutes of ischemia. A, Caspase 2 activity. B, Caspase 3 activity. C, Caspase 8 activity. D, Caspase 9 activity. Closed columns, Control group (n $=6$ for each ischemic time); open columns, PKC regulator-treated group ( $n=6$ for each ischemic time); N.S., not significant. Values obtained from naive hearts of PVG rats (control; no ischemia) served as a reference $(n=4)$. Mean \pm SE values are shown.

our next step was to determine the amount of cardiomyocyte apoptosis in cardiac allografts. Four hours after reperfusion, the number of apoptotic cardiomyocytes increased 2.2-fold in the control group subjected to 120 minutes of ischemia as compared with the control group subjected to 30 minutes of ischemia. In addition, the PKC regulator treatments reduced the number of apoptotic cardiomyocytes in cardiac allografts subjected to 120 minutes of ischemia by approximately $40 \%$, similar to the reduction observed in cardiac allografts subjected to only 30 minutes of ischemia (Figure $1, B)$. Paralleling the increased number of apoptotic cardiomyocytes, caspase 2, 3, 8, and 9 activities were significantly increased in the control group subjected to 120 minutes of ischemia as compared with the 30-minute ischemic controls (4.5-, 4.1-, 2.1-, and 2.4-fold increase in caspase 2, 3 , 8, and 9, respectively; Figure 2). After 30 minutes of ischemia, caspase 9 activity-but not caspase 2,3 , or 8 activity-significantly decreased in the PKC regulatortreated group as compared with the control group (Figure 2). However, we found that PKC regulator treatments decreased caspase 2, 3, and 9 activity by $42 \%, 88 \%$, and $67 \%$, respectively, but not caspase 8 activity, as compared with the 120-minute ischemic controls (Figure 2). Furthermore, treatment with PKC regulators reduced caspase 2, 3, and 9 activity in the 120-minute ischemic group to the levels found in hearts subjected to only 30 minutes of ischemia. Therefore, in the transplanted heart subjected to prolonged ischemia, treatment with $\epsilon \mathrm{PKC}$ activator and $\delta \mathrm{PKC}$ inhibitor reduces caspase 2- and 9-mediated cardiomyocyte apoptosis to the levels observed after only a short ischemic period.

\section{Treatment With PKC Regulators During Prolonged} Ischemia Reduces the Resultant Proinflammatory Response of Ischemia-Reperfusion Injury

Ischemia-reperfusion injury also produces a proinflammatory environment that includes an influx of injurious cytokines and chemokines. ${ }^{5}$ To determine whether treatment with PKC regulators reduces the inflammatory response during prolonged ischemia, we examined neutrophil-produced MPO. Neutrophils are predominant effector cells in the local inflammatory response. ${ }^{18} \mathrm{We}$ also determined the levels of the proinflammatory cytokines and chemokines TNF- $\alpha$, IL- $1 \beta$, and MCP- 
A

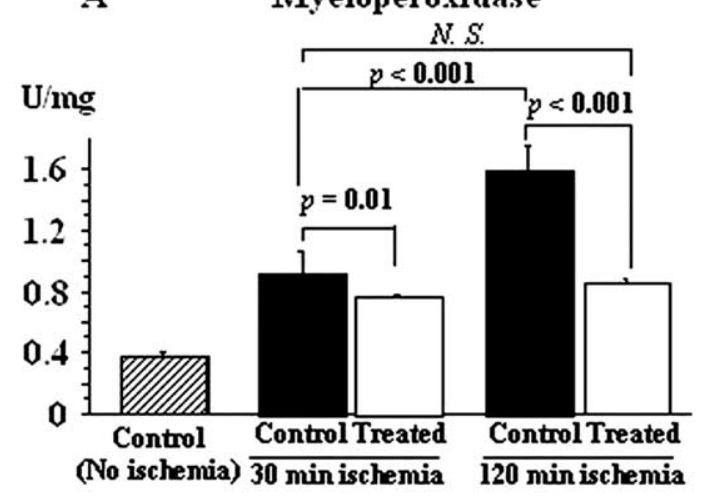

$\mathrm{C}$

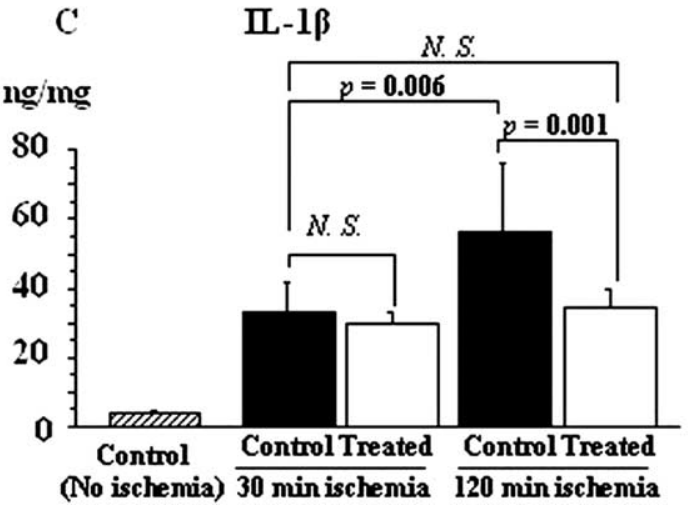

B

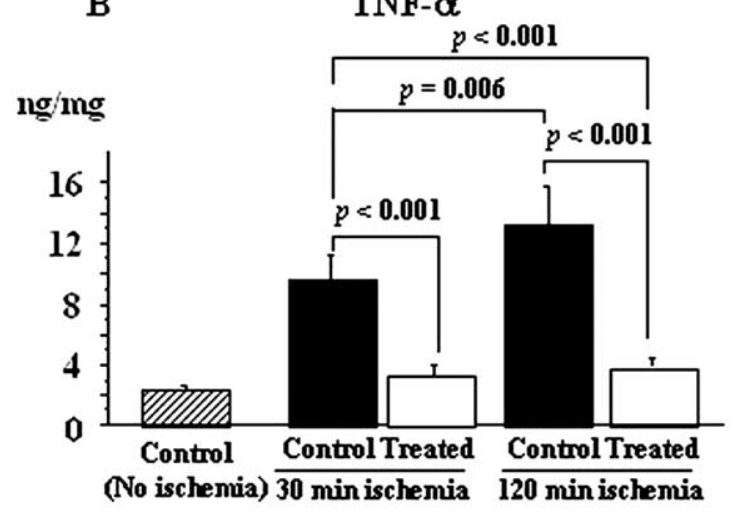

$\mathrm{D}$

MCP-1

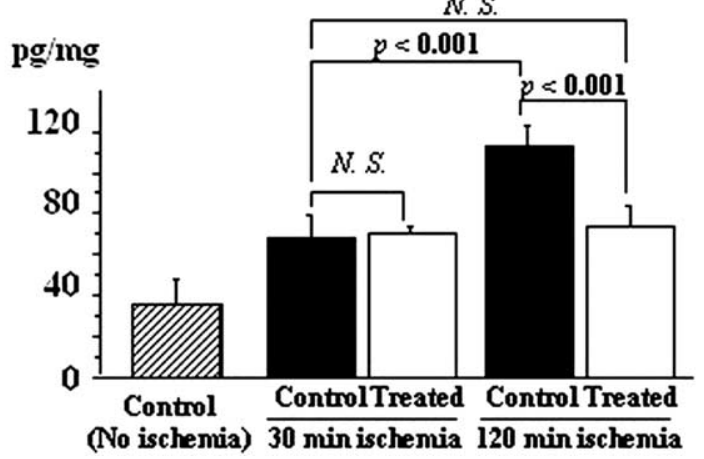

Figure 3. Selective PKC regulators reduce myeloperoxidase (MPO) activity and proinflammatory cytokine production induced by ischemia-reperfusion injury to transplanted hearts subjected to 30 or 120 minutes of ischemia. A, MPO activity. B, TNF- $\alpha$ production. C, IL-1 $\beta$ production. D, MCP-1/CCL-2 production. Closed columns, Control group ( $n=6$ for each ischemic time); open columns, PKC regulator-treated group ( $\mathrm{n}=\mathbf{6}$ for each ischemic time); N.S., not significant. Values obtained from naive hearts of PVG rats (control; no ischemia) served as a reference $(\mathbf{n}=$ 4). Mean \pm SE values are shown.

1/CCL-2. Four hours after transplantation, the levels of MPO and the tested proinflammatory cytokines increased by 1.7 -fold in the 120-minute ischemic controls compared with the 30minute ischemic controls. The production of TNF- $\alpha$ significantly decreased in both PKC regulator-treated groups, whereas the production of IL- $1 \beta$ and MCP-1 decreased ( $41 \%$ and $35 \%$, respectively) only in the 120 -minute ischemic, PKC regulator-treated group (Figure 3). It is important to note that there were no significant differences in these inflammatory responses between the PKC regulator-treated group subjected to 120 minutes of ischemia and the 30-minute ischemic control group (Figure 3). In addition to reducing caspase 2 and 9, PKC regulator treatment during prolonged ischemia also reduces cardiomyocyte apoptosis by minimizing the proinflammatory response, as measured by the reduction in cytokine levels.

Treatment With PKC Regulators Significantly Inhibits GCAD Stimulated by Prolonged Ischemia

There was no early graft loss due to acute rejection until 90 days after transplantation. We first demonstrated that pro- longed ischemia during organ procurement increases GCAD, as measured 90 days after transplantation (Figure 4). A 3.2fold increase in the luminal narrowing percentage, a 4.5 -fold increase in the intima-media ratio, and a 2.5 -fold increase in the diseased vessel percentage were evident. Conversely, treatment with PKC regulators during organ procurement and at reperfusion inhibited GCAD in the cardiac allografts subjected to 120 minutes of ischemia, decreased the percentage of luminal narrowing by $78 \%$, decreased the intima-media ratio by $58 \%$, and decreased the percentage of diseased vessels by $68 \%$ 90 days after transplantation (Figure 4).

\section{Discussion}

In this study, we examined the acute consequences of $\epsilon \mathrm{PKC}$ and $\delta \mathrm{PKC}$ treatment during brief (30 minutes) or prolonged (120 minutes) procurement ischemia and the chronic results on the development of GCAD. These PKC regulators reduce ischemia-reperfusion injury by 2 distinct means: an $\epsilon \mathrm{PKC}$ activator delivered before and early during ischemia 


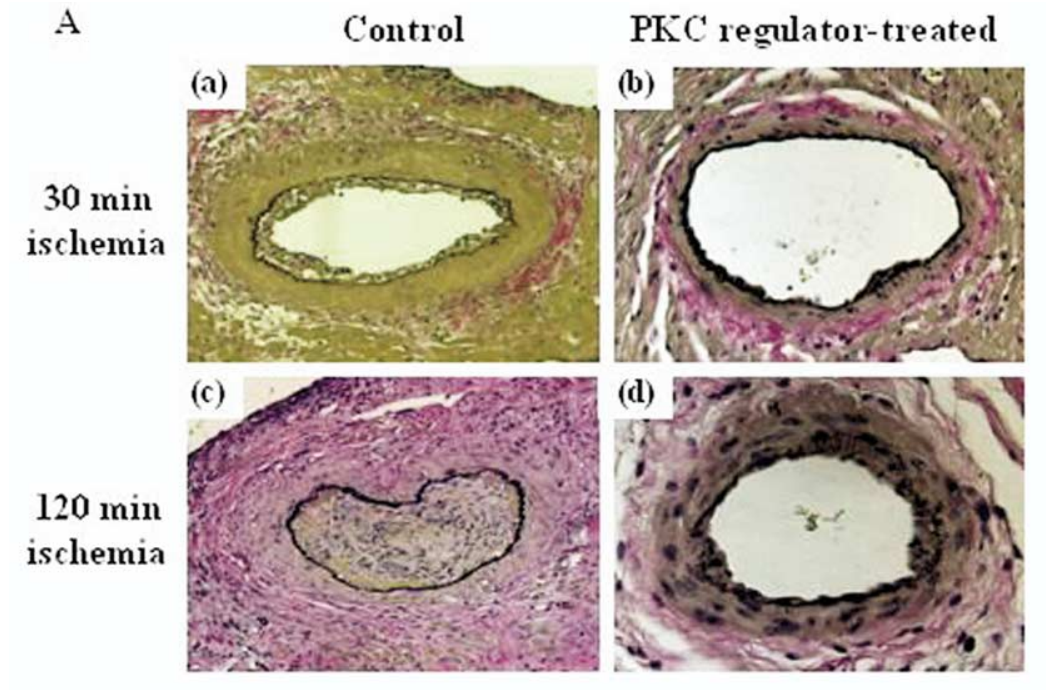

PKC regulator-treated

B

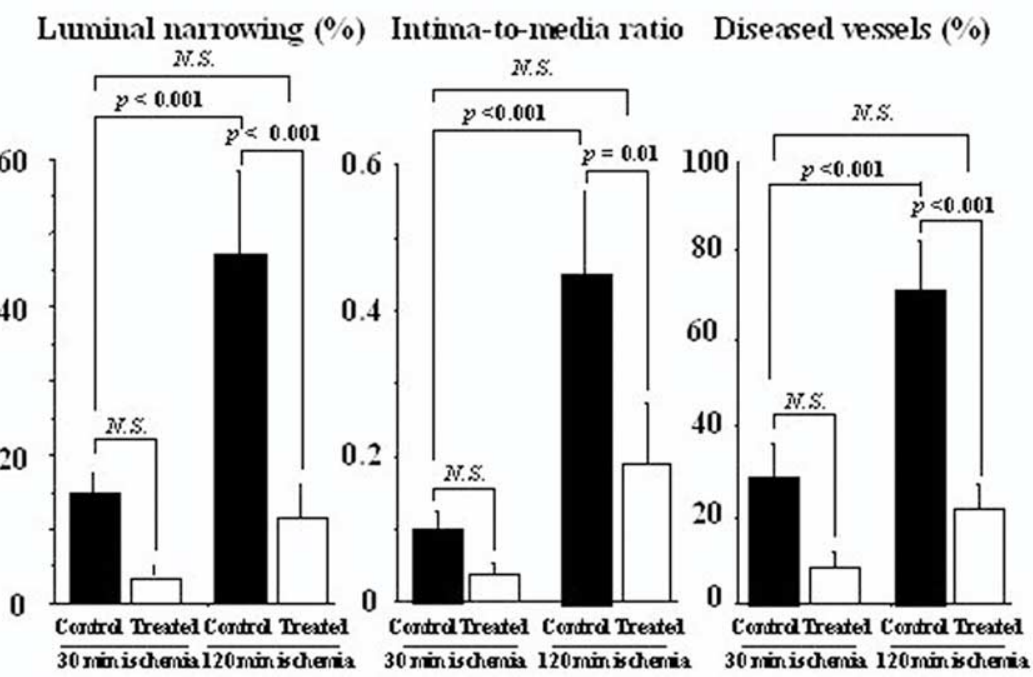

Figure 4. Selective PKC regulators reduce GCAD in cardiac allografts subjected to 30 or 120 minutes of ischemia. A, Representative sections of cardiac allografts stained with Elastica Van Gieson for evaluation of GCAD in the control group with 30 minutes of ischemia (a), the PKC regulator-treated group with 30 minutes of ischemia (b), the control group with 120 minutes of ischemia (c), and the PKC regulator-treated group with 120 minutes of ischemia (d). Note that marked fibrointimal thickening and luminal narrowing, morphologically resembling typical human GCAD, were observed in the control group with 120 minutes of ischemia. In contrast, less intimal thickening and preserved vessel lumen were observed in the control group with $\mathbf{3 0}$ minutes of ischemia and the PKC regulator-treated groups with $\mathbf{3 0}$ and $\mathbf{1 2 0}$ minutes of ischemia. B, Morphometric assessment of cardiac allografts. Note that compared with parameters in the control group with 120 minutes of ischemia, all 3 GCAD parameters were significantly reduced in the PKC regulator-treated group with 120 minutes of ischemia at 90 days after transplantation. In addition, The GCAD indexes of the PKC regulator-treated group with 120 minutes of ischemia did not differ significantly from those of the control group with $\mathbf{3 0}$ minutes of ischemia. Closed columns, Control group ( $n=8$ for each ischemic time); open columns, PKC regulator-treated group ( $n=6$ for each ischemic time); N.S., not significant. Mean \pm SE values are shown.

mimics ischemic preconditioning, ${ }^{10,19}$ and a $\delta \mathrm{PKC}$ inhibitor delivered at reperfusion has an antiapoptotic effect. We found that in control animals, 120 minutes of ischemia increased cardiomyocyte apoptosis; caspase 2, 3, 8, and 9 activity; inflammatory cytokine production; and neutrophil infiltration into the allografts as compared with 30 minutes 
of ischemia. We also found that aggravated cardiac damage with prolonged ischemia correlated with increased GCAD measured 90 days after transplantation. In contrast, combined treatment with the $\delta \mathrm{PKC}$-specific inhibitor $\delta \mathrm{V} 1-1$ and the $\epsilon \mathrm{PKC}$-specific activator $\Psi \in \mathrm{RACK}$ significantly suppressed ischemia-reperfusion injury and resulted in an approximately $70 \%$ reduction in GCAD. The protective effect of these PKC regulators was more significant in the prolonged-ischemia group. In addition, treatment with these PKC regulators reduced all the parameters related to ischemia-reperfusion injury and GCAD after prolonged ischemia to the level of those observed after the short period of ischemia. The PKC regulator-treated group with prolonged ischemia showed an approximately $70 \%$ decrease in the production of the proinflammatory cytokine TNF- $\alpha$ and an approximately $40 \%$ decline in IL- $\beta$ and MCP-1/CCL-2. Significant decreases were also observed in MPO activity, cardiomyocyte apoptosis, and caspase 2, 3, and 9 activity. It is important to note that the reduction in ischemia-reperfusion injury observed 4 hours after transplantation correlated with a reduction in the development of GCAD; there was a $70 \%$ decline in disease severity in the PKC regulatortreated group as compared with the control group.

Activation of $\delta \mathrm{PKC}$ is associated with inhibition of proliferation, cell-cycle arrest, enhanced differentiation, and/or accelerated apoptosis in several nonheart cell lines. ${ }^{20}$ Zhao and associates ${ }^{6}$ have shown that the number of apoptotic cells is increased during reperfusion, that apoptosis may be accelerated by the process of reperfusion, and that inhibition of $\delta \mathrm{PKC}$ reduces reperfusion injury to the myocardium by inhibiting apoptosis. ${ }^{21}$ Our previous study with a porcine model of acute myocardial infarction demonstrated that the cardioprotective effects of $\delta \mathrm{PKC}$ inhibitor may result from inhibiting cell signaling, thereby implicating this isozyme in mediating apoptosis. ${ }^{19}$ In this study, caspase 3 and 9 activity was significantly reduced in the PKC regulator-treated group with prolonged ischemia, whereas no significant reduction in caspase 8 was observed in PKC regulator-treated group after 30 and 120 minutes of ischemia. Thus, apoptosis in the cardiac allograft was reduced mainly by inhibition of the caspase 9-mediated proapoptotic pathway.

Oxygen-derived free radicals are directly implicated in pathologic apoptosis. ${ }^{22}$ We observed a significant increase in both superoxide production and cardiomyocyte apoptosis with prolonged ischemia in comparison to that with a short ischemic period. However, there was no significant reduction in superoxide production in the PKC regulator-treated group in comparison to the saline-treated control group subjected to either 30 or 120 minutes of ischemia. This suggests that this early burst of superoxide production is independent of $\delta \mathrm{PKC}$ and $\epsilon \mathrm{PKC}$.

The role of caspase 2 in apoptosis is still unclear, ${ }^{23}$ but caspase 3 activation by caspase 2 has been reported to involve caspase 9 activation. ${ }^{24}$ We observed a significant increase in caspase 2, 3, and 9 activity after prolonged ischemia, and this was significantly decreased after PKC regulator treatment. This is the first report that shows a suppressive effect by selective PKC regulators on caspase 2 activity in an in vivo heart transplantation model. However, the mechanistic basis for interactions of $\delta \mathrm{PKC}$ and $\epsilon \mathrm{PKC}$ isozymes and caspase 2 activation, and interactions of caspase 2 and downstream caspases, in our experimental model remains to be elucidated.

Most importantly, GCAD was significantly reduced at 90 days after transplantation after PKC regulator treatment. This reduction after 120 minutes of ischemia was comparable to the level of GCAD after 30 minutes of ischemia. Because the half-life of the PKC-regulation peptides is very short, the reduction in GCAD that we observed after the 120-minute ischemia most likely occurred because of the acute reduction in ischemia-reperfusion injury in the early phase. The early cell-protective effect might have resulted in decreased production of proinflammatory cytokines in the cardiac allograft, which in turn led to a decreased GCAD.

Although this study used an allogenic model of heart transplantation as a model of cardiac transplantation in humans, the ischemic period of 120 minutes is shorter than the mean ischemic time in humans (3.1 hours). ${ }^{1}$ Nevertheless, GCAD progression in the rodent model is accelerated relative to that in humans ${ }^{16}$ and therefore may be useful to begin assessing new therapies to prolong graft survival. In addition, this model examined sequential treatment of the donor heart with the $\epsilon \mathrm{PKC}$ activator in the cardioplegic solution followed by treatment with the $\delta \mathrm{PKC}$ inhibitor in the recipient rat just before reperfusion. The study did not determine the optimal timing of treatment or the concentration of these peptides to obtain optimal protection of GCAD. Although these PKC regulators did not exert significant effects on most of the measured parameters as compared with the saline-treated controls, in allografts subjected to a short ischemic insult (30 minutes), there were trends of reduction in ischemia-reperfusion injury and GCAD in the PKC regulator-treated group as compared with saline-treated controls. The differences between the PKC regulator-treated group and the saline-treated controls became more apparent with increased ischemia-reperfusion injury and GCAD caused by prolonged ischemia (120 minutes).

Because the goal for this study was to design a clinically relevant protocol, the side effects of the $\delta \mathrm{PKC}$ inhibitor, which was delivered systemically to the recipient before reperfusion, should be discussed. Previous study has shown that a single bolus dose of $\delta \mathrm{PKC}$ inhibitor $\left(\mathrm{TAT}_{47-57} /\right.$ $\delta \mathrm{V} 1-1)$ administered by intracoronary delivery in pigs (5 $\mu \mathrm{g} / \mathrm{kg}$ ) resulted in no toxic effects in the kidney, spleen, liver, or lung. ${ }^{19}$ Furthermore, $\delta \mathrm{PKC}$ knockout mice are 
viable and have a very subtle phenotype associated with free-radical formation in the vein graft response (there was a decrease in this damage), ${ }^{10}$ thus supporting our observation on the nontoxic effect of $\delta \mathrm{PKC}$ inhibitor, but the benefit for vasculopathy in the graft.

In conclusion, our results suggest that GCAD—and possibly organ failure induced by prolonged ischemia of the donor heart - may be inhibited by combined treatment with an $\epsilon \mathrm{PKC}$ activator and a $\delta \mathrm{PKC}$ inhibitor in clinical cardiac transplantation.

\section{References}

1. Taylor DO, Edwards LB, Mohacsi PJ, Boucek MM, Trulock EP, Keck $\mathrm{BM}$, et al. The registry of the International Society for Heart and Lung Transplantation: twentieth official adult heart transplant report-2003. J Heart Lung Transplant. 2003;22:616-24.

2. Vassalli G, Gallino A, Weis M, von Scheidt W, Kappenberger L, von Segesser LK, et al. Alloimmunity and nonimmunologic risk factors in cardiac allograft vasculopathy. Eur Heart J. 2003;24:1180-8.

3. Gaudin PB, Rayburn BK, Hutchins GM, Kasper EK, Baughman KL, Goodman SN, et al. Peritransplant injury to the myocardium associated with the development of accelerated arteriosclerosis in heart transplant recipients. Am J Surg Pathol. 1994;18:338-46.

4. Wang CY, Aronson I, Takuma S, Homma S, Naka Y, Alshafie T, et al. cAMP pulse during preservation inhibits the late development of cardiac isograft and allograft vasculopathy. Circ Res. 2000;86:982-8.

5. Bergese SD, Huang EH, Pelletier RP, Widmer MB, Ferguson RM, Orosz CG. Regulation of endothelial VCAM-1 expression in murine cardiac grafts. Expression of allograft endothelial VCAM-1 can be manipulated with antagonist of IFN-alpha or IL-4 and is not required for allograft rejection. Am J Pathol. 1995;147:166-75.

6. Zhao ZQ, Nakamura M, Wang NP, Wilcox JN, Shearer S, Ronson RS, et al. Reperfusion induces myocardial apoptotic cell death. Cardiovasc Res. 2000;45:651-60.

7. Nishizuka Y. Studies and perspectives of protein kinase C. Science. 1986;233:305-12.

8. Dorn GW II, Souroujon MC, Liron T, Chen CH, Gray MO, Zhou HZ, et al. Sustained in vivo cardiac protection by a rationally designed peptide that causes epsilon protein kinase C translocation. Proc Natl Acad Sci U S A. 1999;96:12798-803.

9. Chen L, Hahn H, Wu G, Chen CH, Liron T, Schechtman D, et al. Opposing cardioprotective actions and parallel hypertrophic effects of delta PKC and epsilon PKC. Proc Natl Acad Sci U S A. 2001;98: 11114-9.
10. Inagaki K, Hahn HS, Dorn GW II, Mochly-Rosen D. Additive protection of the ischemic heart ex vivo by combined treatment with delta-protein kinase $\mathrm{C}$ inhibitor and epsilon-protein kinase $\mathrm{C}$ activator. Circulation. 2003;108:869-75.

11. Tanaka M, Terry RD, Mokhtari GK, Inagaki K, Koyanagi T, Kofidis $\mathrm{T}$, et al. Suppression of graft coronary artery disease by a brief treatment with a selective epsilonPKC activator and a deltaPKC inhibitor in murine cardiac allografts. Circulation. 2004;110(11 suppl 1):II194-9

12. Ono K, Lindsey ES. Improved technique of heart transplantation in rats. J Thorac Cardiovasc Surg. 1969;57:225-9.

13. Uemura S, Matsushita H, Li W, Glassford AJ, Asagami T, Lee KH, et al. Diabetes mellitus enhances vascular matrix metalloproteinase activity: role of oxidative stress. Circ Res. 2001;88:1291-8.

14. Chen Z, Chua CC, Ho YS, Hamdy RC, Chua BH. Overexpression of $\mathrm{Bcl}-2$ attenuates apoptosis and protects against myocardial I/R injury in transgenic mice. Am J Physiol Heart Circ Physiol. 2001;280: H2313-20.

15. Mullane KM, Kraemer R, Smith B. Myeloperoxidase activity as a quantitative assessment of neutrophil infiltration into ischemic myocardium. J Pharmacol Methods. 1985;14:157-67.

16. Tanaka M, Fedoseyeva EV, Robbins RC. Graft coronary artery disease in murine cardiac allografts: proposal to meet the need for standardized assessment. J Heart Lung Transplant. 2005;24:316-22.

17. Hess ML, Kukreja RC. Free radicals, calcium homeostasis, heat shock proteins, and myocardial stunning. Ann Thorac Surg. 1995;60:760-6.

18. Zimmerli W, Lew PD, Waldvogel FA. Pathogenesis of foreign body infection. Evidence for a local granulocyte defect. J Clin Invest. 1984;73:1191-200.

19. Inagaki K, Chen L, Ikeno F, Lee FH, Imahashi K, Bouley DM, et al. Inhibition of delta-protein kinase $\mathrm{C}$ protects against reperfusion injury of the ischemic heart in vivo. Circulation. 2003;108:2304-7.

20. Mischak H, Goodnight JA, Kolch W, Martiny-Baron G, Schaechtle C, Kazanietz MG, et al. Overexpression of protein kinase C-delta and -epsilon in NIH 3T3 cells induces opposite effects on growth, morphology, anchorage dependence, and tumorigenicity. J Biol Chem. 1993;268:6090-6.

21. Heidkamp MC, Bayer AL, Martin JL, Samarel AM. Differential activation of mitogen-activated protein kinase cascades and apoptosis by protein kinase $\mathrm{C}$ epsilon and delta in neonatal rat ventricular myocytes. Circ Res. 2001;89:882-90.

22. Greenlund LJ, Deckwerth TL, Johnson EM Jr. Superoxide dismutase delays neuronal apoptosis: a role for reactive oxygen species in programmed neuronal death. Neuron. 1995;14:303-15.

23. Troy CM, Shelanski ML. Caspase-2 redux. Cell Death Differ. 2003; 10:101-7.

24. Robertson JD, Enoksson M, Suomela M, Zhivotovsky B, Orrenius S. Caspase-2 acts upstream of mitochondria to promote cytochrome $\mathrm{c}$ release during etoposide-induced apoptosis. J Biol Chem. 2002;277: 29803-9. 